\title{
Regard sur le passé de la géotechnique
}

\section{J.-L. BORDES}

20, rue de Madrid 75008 Paris
Dans l'esprit d'une rétrospective propre à la fin du siècle, on s'est proposé de regarder l'histoire de la géotechnique, bien connue par ailleurs, en considérant tout spécialement l'évolution de ses pratiques expérimentales et de la notion de laboratoire depuis le $\mathrm{XVII}^{*}$ jusqu'à la première moitié du $\mathrm{XX}^{*}$ siècle. La relation de leur développement avec les progrès des connaissances, et l'amélioration des procédés de construction, est tout particulièrement soulignée. En même temps, on a mis en évidence l'ancienneté et l'importance de l'augmentation des moyens de diffusion du savoir, livres et publications, sociétés savantes, des différents modes de transferts technologiques au nombre desquels les voyages et les congrès, tous d'autant plus efficaces que la formation initiale des hommes par l'école a été bien faite.

\section{Looking back over the history of geotechnics}

but we focus here on the evolution of experimental practice and the very notion of laboratories from the 18 th century through to the middle of the 20th. Particular stress is put on the relationship between their development as knowledge improved and the improvement of construction methods. In so doing, we also underscore the importance - and indeed the very age of the practice - of making knowledge more widely available, through books, magazines, and learned societies, and of the different methods of transferring technological know-how, through trips and conferences. All these methods of enhancing knowledge have always derived the greatest benefit from the soundest basic academic grounding. 


\section{Introduction}

S'intéresser au passé de la géotechnique, alors qu'il s'agit de célébrer le passage d'un millénaire à un autré, et d'esquisser à cette occasion des perspectives de progrès et de développement peut paraître paradoxal. Il n'en est rien, l'avenir ne prend toute sa signification qu'à la lumière du passé. Pour savoir où aller, encore faut-il connaitre où l'on est, et d'où l'on vient. Nous nous proposons de rappeler brièvement ces origines, en insistant sur quelques faits en rapport avec la situation présente et celle que nous pouvons imaginer pour l'avenir.

L'ensemble des disciplines constituant le génie civil a été élaboré à partir du XVIII siècle, mais la mécanique des sols n'a été constituée en discipline à part entière qu'à partir des travaux de K. Terzaghi dans les années 20, et pleinement reconnue que depuis son premier congrès international tenu aux USA, à Cambridge, en 1936

Il s'était pourtant passé beaucoup de choses avant. Les hommes avaient utilisé la terre comme support de leurs constructions ou comme matériaux constituant ces mêmes constructions avec ou sans addition d'autres matériaux tels que la pierre ou le bois, depuis des millénaires. Mais la modélisation du comportement de ces structures n'a guère commencé qu'au XVHI siècle, et il est d'usage de considérer que le mémoire de Coulomb est le document qui marque le début d'un processus de conceptualisation du comportement des sols qui continue sous nos yeux

Cette histoire a déjà fait l'objet de publications nombreuses $^{\text {h1) }}$. Aussi nous souhaiterions ne pas recommencer un récit qui a été très bien fait par d'autres, et que nous utiliserons abondamment dans la suite de cet exposé

Il nous a semblé intéressant de relire cette histoire en considérant celle des essais en place et en laboratoire et de l'observation des ouvrages, de façon à mettre en évidence leur articulation avec les méthodes de calculs qui traduisaient en langage mathématique les modélisations du comportement des structures. A cette histoire, nous rajoutons celle de la diffusion des connaissances, entre les différentes communautés techniques.

Si on laisse de côté toute la période de l'Antiquité du Moven Age et de la Renaissance, en suivant l'analyse qui en a été faite par Skempton, on peut distinguer quatre périodes, auxquelles nous ajouterons celle qui couvre le $\mathrm{XX}^{\mathrm{e}}$ siècle après les travaux de Terzaghi:

\footnotetext{
(1) On se reportera tout particulièrement aux comptes rendus du $11^{*}$ Concrès international de mécanicue des sols et des travaux de fondations à San Francisco, tenu du 12 au 16 aout 1985. Le volume du Jubilề sous le titre, Le génie géotechnique, des origines à nos jours. rassemble les textes des conférences prononcées par Jean Kérisel sur 1. Thistoire de lingénierie géomècanique jusqu'à 1700 p. A.W. Skempton sur \& l'évolution de la connaissance des sols de 1717 à 1927 D. Ralph B. Peck sur a les soixante dernières années

Quatre autres publications sont à mentionner:

Habib P. - a Histoire de la mécanique des sols n. Cahiers de rhêologie, mars 1991.

Kerisel J. - k Historique de la mécanique des sols en France jusqu'al xxt siècle n. Géotechnique vol 6. 1956

Skempton A.W. - $\alpha$ Landmarks in early soil mechanics $x$. Proceedings 7 th European conference on soils mechanics, Brighton, 1979.

Timoshenko S.P. - History of strength of materials. New York Mc Graw Hill, 1953. Réédité depuis 1982 par Dover publications, ine. New York
}

(i) la période préclassique coincidant avec le Xvin' siècle:

(ii) la première phase de la mécanique des sols classique inaugurée par Coulomb en 1774 ,

(iii) la seconde phase à partir des travaux de Darcy en 1856 ;

(iv) la première phase de la mécanique des sols moderne (1910-1927), à partir des travaux d'Atterberg :

(v) la deuxième phase de la mécanique des sols modernes à partir des résultats des travaux de Terzaghi, qui s'étend jusqu'à nos jours.

Or, chacune de ces périodes a été marquée par d'importants travaux expérimentaux qui ont précédé ou accompagné l'élaboration des théories qui constituaient les outils nécessaires aux ingénieurs dans leur travail de conception et de réalisation des ouvrages. Aussi suivra-t-on la mise en place des pratiques et des structures d'études et de recherche qui, au fur et à mesure de l'avancement des connaissances, ont permis le recueil des mesures et observations sans lesquelles aucun progrès n'était possible.

En parallèle avec la division proposée par Skempton, on distinguera un temps d'avant les laboratoires qui couvre à peu près les deux premières parties, celle des laboratoires d'étude des matériaux qui comprend les deux parties suivantes, et enfin une troisième période qui commence après les travaux de Terzaghi. Par laboratoire, nous entendons la structure dans le sens le plus large du terme, dans laquelle peut se développer l'ensemble des pratiques expérimentales qui comprennent les essais sur échantillon, les essais sur modèle, les essais in situ, ainsi que le recueil des observations sur le comportement des ouvrages ou parties d'ouvrages.

\section{Le temps d'avant les laboratoires (jusqu'au milieu du XIX ${ }^{e}$ siècle)}

Ce temps englobe à la fois celui de la période préclassique et de la première phase de la mécanique des sols classique. La période préclassique couvre la plus

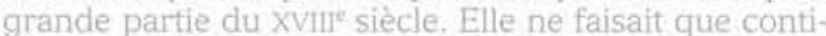
nuer le siècle précédent par bien des aspects. Au cours de cette période, furent élaborées des théories empiriques sur la poussée des terres, fondées sur l'observation. Fixer un commencement est toujours délicat. Il en est ainsi de la première phase de la mécanique des sols classiques. Celui dont la postérité retient le nom, sans vouloir en diminuer les mérites, s'inscrit dans la conti nuité de l'accumulation d'un savoir auquel il va faire faire un bond à partir d'une analyse et d'une synthèse qu'il ne doit qu'à son talent, voire son génie. Mais la période à laquelle il intervient, l'urgence des proqrès à faire, les conditions économiques et sociales de son époque, le matériau accumulé par ses prédécesseurs ne sont pas non plus indifférents à sa réussite.

C'est le cas du mémoire de Coulomb (1736-1806) ${ }^{\text {(2) }}$ qui n'a pas surgi du néant mais qui a été nourri d'un ensemble d'observations, d'essais et d'hypothèses. pour lesquels il faut citer les noms de Vauban (1633.

5. Coulomb C.A. - Essal sur une application des rèales de maximis ef minimis, à quelques problèmes de statique, relatifs à l'architecture. Mèmoire des savants étrangers, Académie royale des sciences, vol. 7. Paris, 1776 (pour 1773).

Voir aussi : Heyman 1. - Coulomb's memoir on statics. Cambridge University press, 1972 


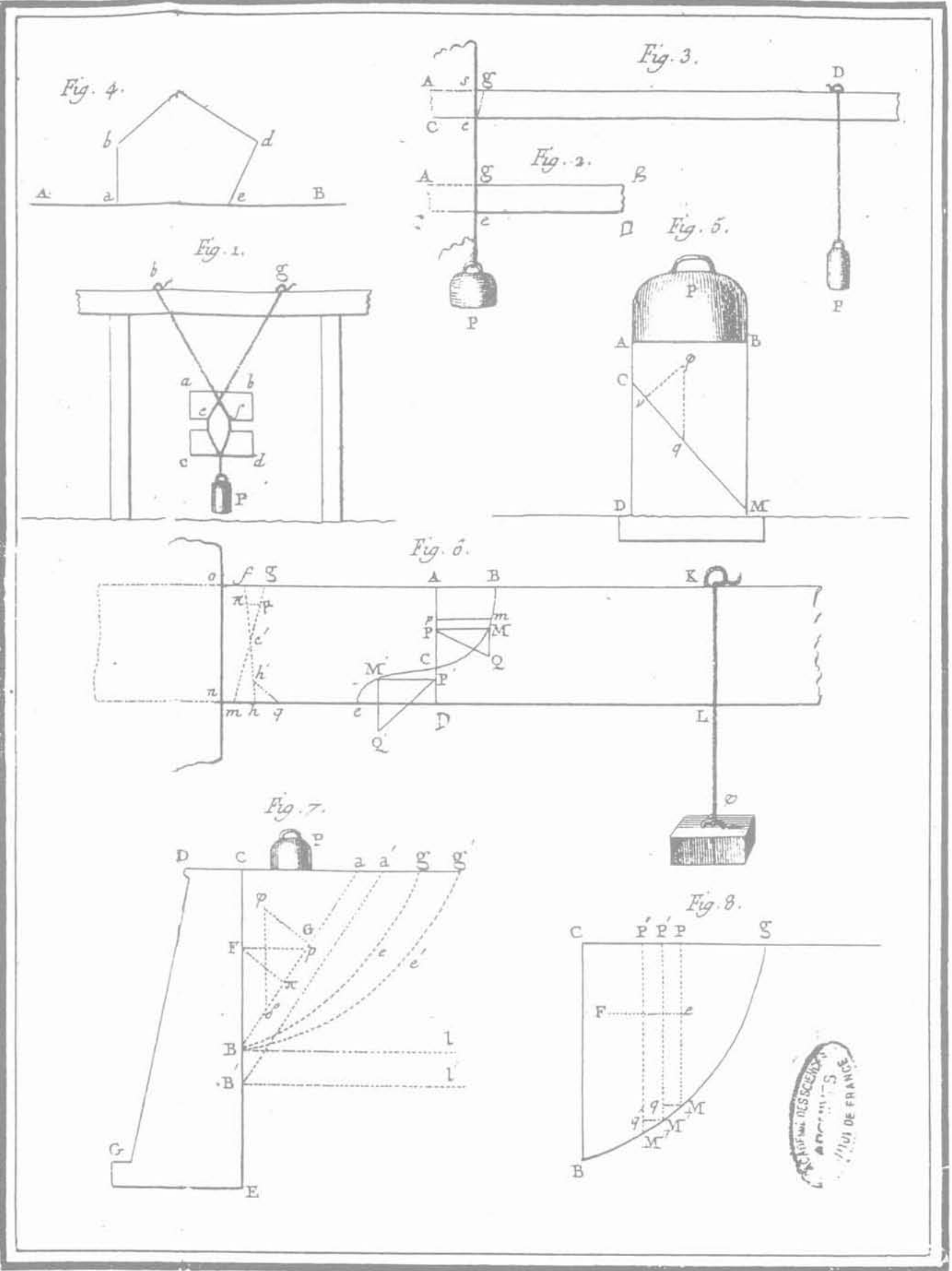

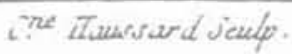

FiG.1 Première planche illustrant le mémoire de Coulomb (bibliothèque de l'Académie des sciences), Coulomb's memoire. 


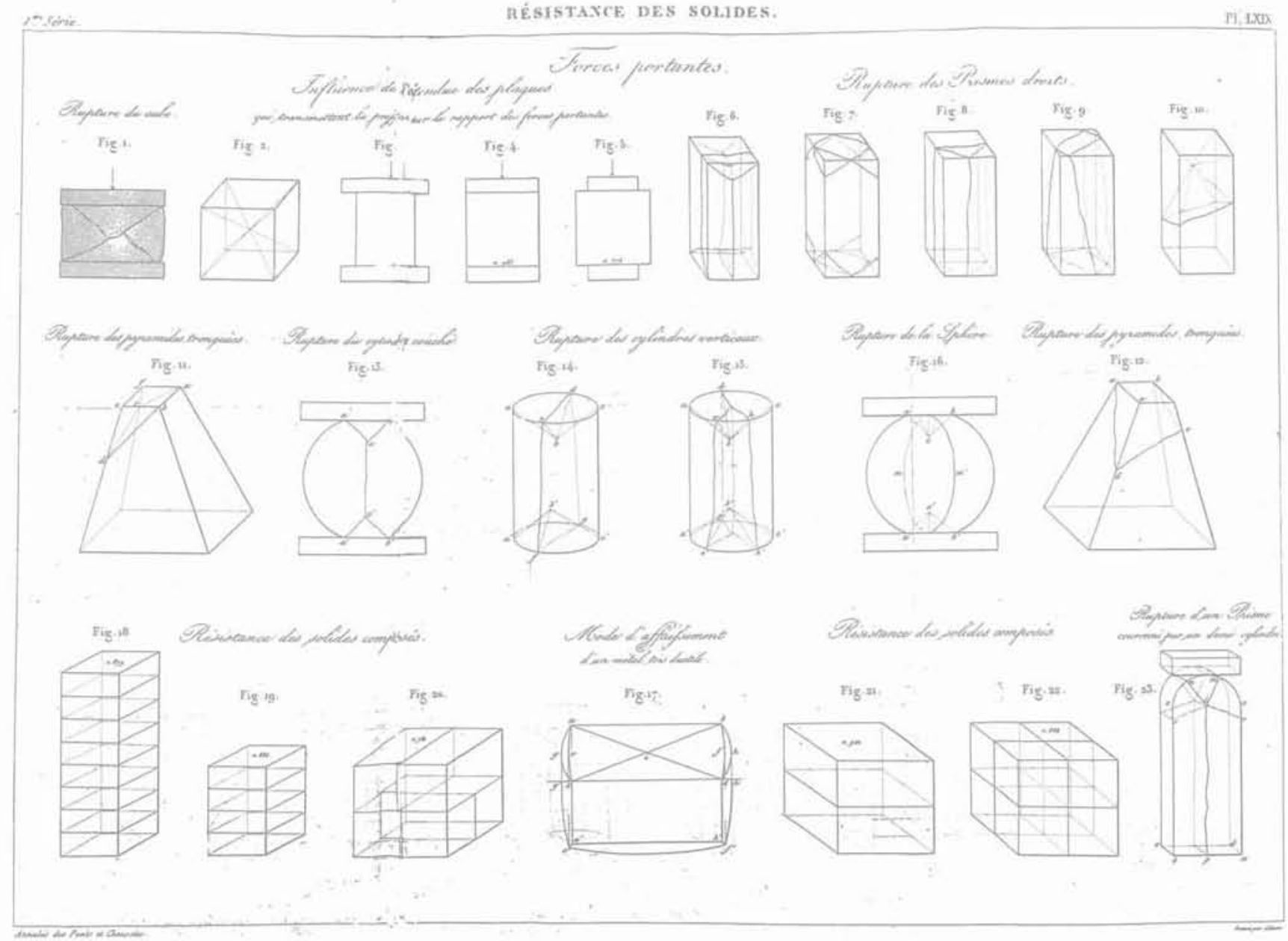

FG, \& Conditions de rupture d'éprouvettes de différentes formes.

Conditions of failure of specimens of various shapes.

1707), Gauthier (1660-1737), Belidor (1694-1761), Gadroy (1705-1759), Perronet (1708-1794), et de l'abbé Bossut (1730-1814), qui fit des essais sur le frottement et la cohésion et fut le professeur de Coulomb à l'École du génie à Mézières, Les notions de prisme de poussée, de talus naturel étaient le fruit d'analyse et de réflexion antérieure. Il n'est pas non plus indifférent que, dès les premières pages de son mémoire, Coulomb se réfère à des essais sur le frottement, faits par Amontons (16631705), rende compte d'essais de mesure sur la cohésion à partir de la rupture de différentes éprouvettes de matériau fait par lui-même (Fig. 1), ou sur un pilier carré par Musschenbroëk (1692-1761). Son analyse s'enracinait dans une approche expérimentale qui était la règle à l'époque. L'importance du mémoire de Coulomb qui dépassait la seule mécanique des sols, mais qui apportait des éléments de réflexion essentiels aussi bien au calcul des voûtes qu'à celle des poutres, était telle qu'il fallut plusieurs dizaines d'années pour qu'il fût assimilé.

A la suite de Coulomb, Prony (1755-1839), par une analyse complémentaire, contribuait à la diffusion de l'apport de Coulomb et à son acceptation en France. Mayniel (1760-1809) réalisait des essais sur modèle de $1,5 \mathrm{~m}$ de haut, Français (1775-1833) introduisait dans le calcul cohésion et fruit du mur de soutènement. Poncelet (1788-1867) établissait les calculs avec talus et mur inclinés, frottement sur le mur et introduisit la notation $\phi$. Navier (1775-1833) donnait des valeurs de l'angle de frottement de matériau pulvêrulent et des observations sur la tenue des talus en argile. En parallèle, ces mèmes ingénieurs s'intéressaient à la mesure de la résistance mécanique de matériau comme la pierre pour la caractériser en vue du choix des matériaux les plus aptes suivant leur utilisation.

E.-M. Gauthey (1732-1806), dans la tradition des ingénieurs du XVIII siècle, dont il est un des plus brillants représentants, qui s'intéressaient essentiellement aux propriétés à la rupture des matériaux de construction, construisit une machine pour écraser des échantillons. Il fit une série d'essais pour le choix des pierres à employer dans les ponts ou dans des constructions exceptionnelles comme le Panthéon.

Vicat (1786-1861) dans un volumineux article ${ }^{(3)}$ en date de 1833, rendait compte d'essais à la rupture d'échantillons de nombreux matériaux, dans lesquels il essayait de déterminer l'influence de la forme des échantillons, le temps d'application de la charge, définissant par la même occasion les résistances instantanée et différée du matériau (Fig. 2). Il s'appuyait sur la définition de la résistance à la traction ou à l'arrachement, la résistance à la compression ou force portante du matériau, et enfin la ténacité ou force transverse

19icat L.J. - $\alpha$ Recherches expérimentales sur les phénomènes physiques qui précèdent et accompagnent la rupture ou l'affaissement d'une certaine classe de solide $n$. Annales des ponts et chaussées, $2^{6}$ semestre 1833 


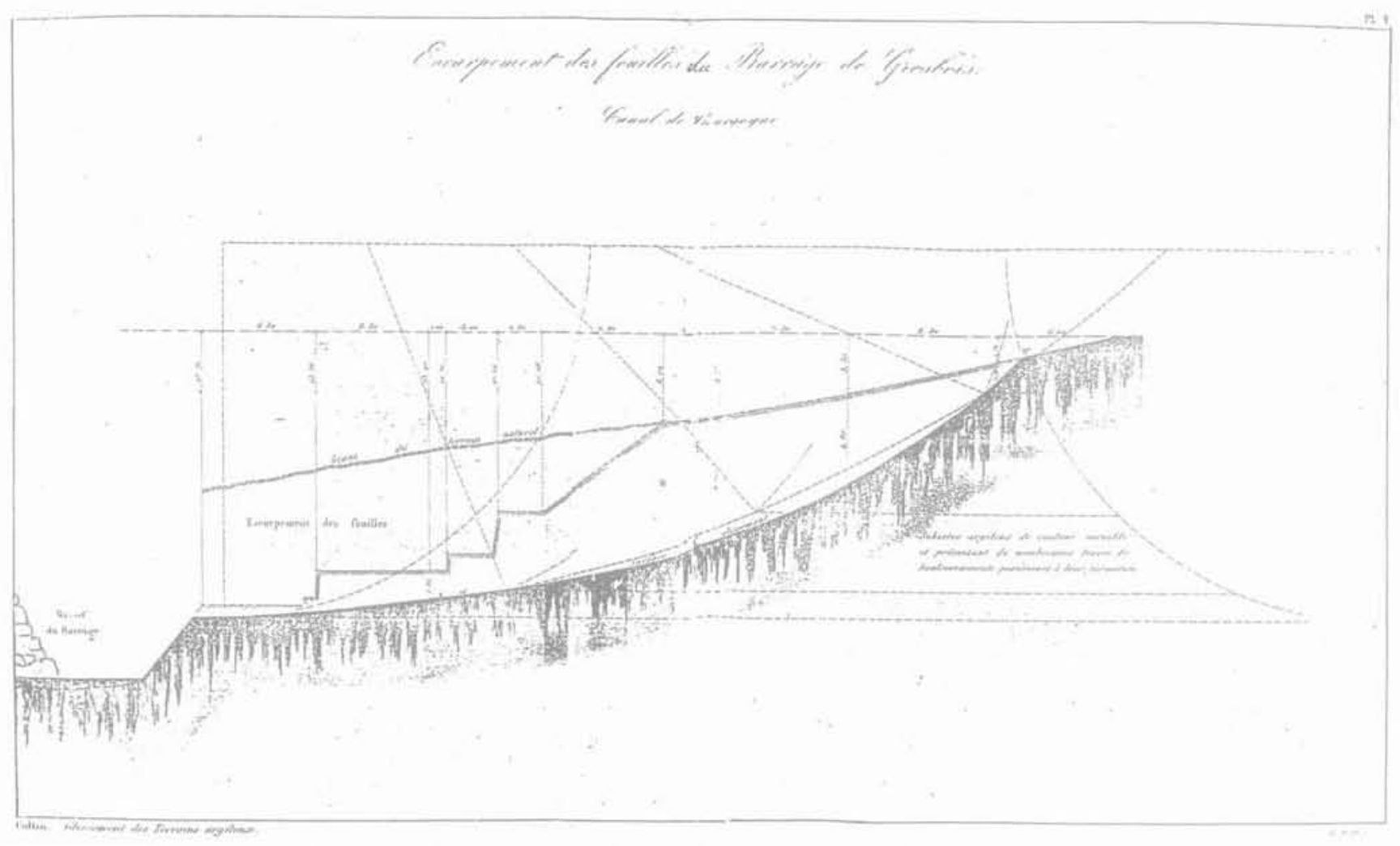

FIG.3 Relevé du glissement survenu en rive droite du barrage de Grosbois. Survey of slide in right bank of Grosbois dam.

dans laquelle intervenait la notion de cohésion de Coulomb. La notion de ténacité appelée à disparaitre, était apparue avec Lemaire (1737). On peut, à la lecture de certaines pages du mémoire de Vicat, y voir l'ébauche de la notion de loi du matériau.

Ces pratiques s'inscrivajent dans une démarche expérimentale qui, par déduction, a permis de bâtir des modèles qui ont été par la suite traduits en langage mathématique. C'était celles des ingénieurs savants du XVIII siècle qui partant de problèmes très concrets posés par l'exercice de leur métier, cherchaient à élaborer une théorie pour résoudre ces problèmes au moindre coùt, et la confrontaient à l'épreuve des faits. Toute cette recherche effectuée par des praticiens, à la fois maitres d'ouvrage et maitres d'ceuvre, s'inscrivait dans le cacire des travaux de fortification qui furent exècutés dans la deuxième moitié du XVII' et le début du $\mathrm{XIX}^{e}$ siècle. Le prestige des travaux antérieurs de Vauban a occulté le fait que le volume des terrassements et des maçonneries, pendant cette dernière période d'environ cinquante ans, fut bien supérieur. La connaissance du mécanisme de la stabilité des murs de soutènement était un enjeu économique de première importance. Les ingénieurs du début du XIX ${ }^{c}$ siècle imprégnés des idées saint-simoniennes, agirent de la même façon, pour remplir au mieux le rôle qu'ils estimaient être le leur dans le cadre des réalisations de la Révolution industrielle.

Pendant la première moitié du XIX $x^{*}$ siècle, les ingénieurs français, à cause de leurs connaissances mathématiques bien supérieures à celle de leurs confrères étrangers, furent occupés par le développement de la théorie de l'élasticité et son application à la résistance des matériaux, domaine dans lequel ils jouèrent un rôle essentiel. Alors que ces théories étaient nées d'une pra- tique expérimentale, on put souvent observer une dérive vers plus de théorie.

De leur côté, les Anglais plus pragmatiques par tradition culturelle, s'intéressèrent aux propriétés mécaniques des matériaux et en particulier du métal. En Grande-Bretagne, la production de fer avait cru de 690500 tornes en 1827 à 3659000 tonnes en 1857 , alors qu'à cette dernière date la production en France n'était que de 586000 tonnes.

Mais Lamé (1795-1870) et Clapeyron (1799-1864), dont la contribution à la théorie de l'élasticité entre 1820 et 1830 , à la suite de Navier, avec Cauchy et Poisson est bien connue, pourraient étre considérés comme les promoteurs du laboratoire d'essais des matériaux, pendant leur séjour comme professeurs à l'école du génje à Saint-Pétersbourg, avant 1830. C'est dans le cadre de mission de conseil pour des ouvrages qu'ils construisirent une machine d'essais.

Un autre exemple de démarche expêrimentale intéressante, c'est celle adoptée par le capitaine Moreau lors d'essais, conduits en 1825 sur des pleux de sable, qui ont donné lieu à des essais de chargement avec des mesures d'enfoncement d'une précision meilleure que le millimètre et sur des périodes d'observation de six mois. Ce fut l'occasion de mettre en évidence les phénomènes d’arc-boutement dans le sable. Niel, à peu près en 1831 , procédait à des mesures à la suite d'améliorations de fondations très médiocres par apport de sable.

Cependant, A. Collin (4) (1808-1890) demeure l'exemple le plus important d'ingénieurs qui dans le

is Skempton A.W. - un pionnier de la mécanique des sols Alexandre Collin (1808-1890) is. Annales des ponts et chausseec, maijuin 1956, traduction de l'article paru dans les transactions de la Newcomen Society, vol. 25, Londres. 1946. 
domaine des sols pratiquait son métier en essayant de s'appuyer sur la méthode expérimentale. Ingénieur des ponts et chaussées, il fut en poste, notamment de 1832 à 1844, au canal de Bourgogne. Il a fait la synthèse des données et observations de ruptures survenues dans cinq digues, sept remblais de canaux, deux talus en déblais de route et 12 tranchées de canaux soit un total de 26 sites. II avait été le témoin de six d'entre elles, l'acteur de l'analyse des causes et le projeteur des réparations. Sur certains, il y avait eu non pas un, mais plusieurs glissements. Il s'inscrivait dans une lignée d'ingénieurs qui comprend Vauban, Perronnet et Girard dont il reprenait opinions et expériences.

Il a réuni de précieuses observations sur le terrain, en notant les formes des glissements, leur position par rapport à différentes couches, les conditions dans lesquelles se sont produites les ruptures. Il s'est intéressé à la détermination des propriétés de l'argile en faisant des essais de cisaillement en laboratoire, en fonction de la teneur en eau et de la vitesse de sollicitation, au moyen d'un appareil conçu par lui. Il est, à ce titre, le premier expérimentateur des sols en laboratoire. Il a fait des remarques sur la stabilité à long terme et sur la stabilité à court terme des talus, et proposé le premier calcul avec $\phi=0$.

Sa démarche s'appuie sur l'élaboration d'une théorie à partir d'une analyse aussi rigoureuse que possible des observations sur le terrain. Dans un autre domaine, c'est ainsi qu'à Grosbois, dans le cadre de l'expertise des désordres ayant affecté le barrage, il a procédé en 1838 à des essais de chargement in situ des argiles de fondations du barrage de Grosbois pour estimer leur déformabilité. Dès 1833, au moyen de puits, pour la première fois, dans l'histoire du génie civil, il avait effectué un relevé de la surface d'un glissement en rive gauche du même barrage (Fig. 3).

A la différence de beaucoup d'ingénieurs de son époque qui avaient tendance à prendre les problèmes en force, A. Collin savait qu'on ne pouvait pas s'opposer à un glissement qui a démarré, et que l'eau intérieure ou celle infiltrée à partir des pluies était à l'origine de la plupart des sinistres par les modifications apportées au comportement de l'argile dans son ensemble. Aussi distinguait-il, sans les opposer, les moyens répressifs et préventifs (réparation de l'accident et prévention) des moyens seulement préventifs, comme contreforts et aqueducs souterrains. Il s'impliqua également dans la mise au point de procédé comme celui de l'injection au coulis de ciment, qu'il appliqua à la consolidation de la maçonnerie du barrage de Grosbois. Les résultats de son travail furent publiés dans un ouvrage en 1846. Collin n'a pas eu la postérité qu'il méritait. $\Pi$ fut redécouvert par les chercheurs anglais en 1945.

Cette première période se termine avec les travaux de Rankine (1820-1872) qui réunit dans son manuel de génie civil tout un ensemble de données sur l'angle de frottement, d'observations sur la stabilité des pentes, de considération sur la valeur de la cohésion, et la tenue à court terme des talus, 11 y appliquait sa théorie du champ de contrainte pour les sols pulvérulents (1857) et donnait les expressions et valeurs des coefficients de poussée et butée bien connus.

\section{Les laboratoires d'études des matériaux (milieu du XIXe-premier quart du $X x^{e}$ siècle)}

La période ainsi définie, regroupe la deuxième phase de la mécanique des sols classique, et la première phase de la mécanique des sols modernes. Elle vit l'exécution d'importants travaux expérimentaux d'abord sur les sables puis sur les argiles. Mais il nous semble important de replacer cette évolution dans l'évolution générale des pratiques de l'art de bâtir.

Malgré toutes les approches expérimentales, dont on vient de parler, ce n'est qu'au milieu du XIX' siècle que furent créés de véritables laboratoires ouverts sur l'industrie et les travaux publics. Il s'agit d'un phénomène général en Europe. L'attention que nous portons à la France ne résulte que de la facilité d'obtenir des informations.

Fin 1851, un laboratoire, spécialement consacré aux essais et analyses des calcaires, chaux, ciments et autres substances employées dans la construction, a été organisé à l'Écolé, alors impériale, des ponts et chaussées. Trois cents échantillons furent testés la première année, 553 la deuxième année, dont la moitié pour des travaux portuaires, ce qui montre à la fois la prédominance de ceux-ci et l'importance de la qualité des mortiers soumis à l'agression de l'eau de mer. Le laboratoire de l'École était le laboratoire du corps et travaillait à la demande aussi pour des clients extérieurs. Il en fut ainsi jusqu'en 1949. Avant la fin du XIX: siècle, il eut des implantations régionales, ou des laboratoires furent aussi installés sur des chantiers importants et longs, comme celui du port de Boulogne.

Le génie militaire se dota de laboratoire peu après. De mème que Vicat ne cessa pas de conduire des essais jusqu'à sa mort, les plus grands fabricants de chaux et ciment, se dotèrent de moyens propres d'essais pour améliorer leurs produits,

En 1854, fut créé un laboratoire d'essais à l'initiative du général Morin (1795-1880), directeur du Conservatoire des arts et métiers ${ }^{101}$. Tresca ${ }^{(1)}(1814-1885)$ en fut la cheville ouvrière, mème si Morin y fut très actif. II s'agissait d'un service d'expertise gratuit, mis au service aussi bien de l'État que des collectivités locales. I comprenait un laboratoire d'hydraulique qui permit á Morin, de diffuser son expertise sur les moteurs hydrauliques. Celle-ci était tout à fait exceptionnelle, et avait été acquise aux cours des années 1830-1840, auprès de Poncelet, au moyen d'essais effectués sur tous les moteurs hydrauliques traditionnels ou perfectionnés en usage dans les manufactures de l'État et chez les constructeurs.

Les comptes rendus d'expériences furent diffusés dans les Annales du Conservatoire, gratuitement afin d'en porter les résultats à la connaissance de tous les services techniques officiels. Elles parurent de 1861 à 1880. Contrairement aux espoirs de Morin, c'est le

\footnotetext{
Si Fontanon C - Le laboratoire expèrimental de mécanique, dans te Conseryatoire national des arts et métiers au cceur de Paris. Délégation à l'action artistique de la ville de Paris, 1994.

i6 Les professeurs du Conservatoire des arts et métiers. Dictionnaire biographique (1794-1955), sous la direction de C. Fontanon et A. GreIon. INRP/CNAM. Paris: 1990 . Notice de Bruno Belhoste.
} 
domaine d'essais des matériaux qui se développa le mieux, partie qui était à la charge directement de Tresca. Entre 1864 et 1869, il mena les expériences sur la déformation des corps solides soumis à une pression élevée, qui permirent à Barré de Saint-Venant (17971886) d'établir les équations fondamentales de la plasticité. L'activité du laboratoire déclina après 1870. La mort de Morin en 1880, précipita la fermeture qui fut effective en 1884.

Dans le cadre du Conservatoire, fut crée dans un esprit un peu différent le Laboratoire national d'essai en 1900. Cette structure avait pour but d'assister les petites et moyennes entreprises. Elle comprenait cing sections : physique, métaux, matériaux, machines, chimie. Il regroupait une soixantaine de personnes. Son activité, à la lecture des rapports d'activité avant la guerre de 1914, était florissante. Dans la section matériaux, 1340 essais de compression traction flexion avaient été exécutés, contre 3340 en 1913.

L'échec du laboratoire de Morin serait dû en partie à une mauvaise adéquation avec les demandes du marché. Il y avait une concurrence avec le laboratoire de I'École des ponts et chaussées. Ce dernier s'occupait des structures en métal et du béton, laissant au laboratoire du Conservatoire le domaine des matériaux industriels comme les briques, Ce fut, une occasion perdue pour la pratique technique française de s'ancrer mieux dans l'utilisation des données expérimentales, quand fleurirent partout en Europe, et en Allemagne tout particulièrement, de nombreux laboratoires dans des structures universitaires mais collaborant très étroitement avec l'industrie. Les besoins ne manquaient pas. Le développement technique de la fabrication et de l'emploi des ciments et du béton, l'accumulation des essais de laboratoire, avant mème l'emploi généralisé du béton armé, exigeajent que des efforts de normalisation fussent faits. Les conséquences de l'absence de normes et références sur les relations entre maitre d'ceuvre et entrepreneur étaient extrêmement dommageables. C'est une des explications du nombre très élevé des résiliations de contrat d'entreprise en cours de travaux.

Au milieu du siècle, commencèrent les premières mesures de suivi du comportement des ouvrages. Il nous semble que le premier serait le barrage de Grosbois à partir de fin 1852. Il s'agissait d'un suivi topographique du mouvement de la crête au moyen d'une lunette spécialement prêtée par le laboratoire des ponts et chaussées nouvellement créé. Lors du premier congrès des grands barrages, à Stockholm, en 1933, A. Coyne (1891-1960) analysait les courbes qui furent établies pour les années 1852 à 1861 et reprises après la catastrophe de Bouzey en 1895 jusqu'en 1905 au moins.

Mais le marché de ce type d'ouvrages de génie civil était un tout petit marché. Aussi n'est-il pas étonnant que la mesure du comportement des ouvrages ait surtout été mise en ceuvre pour les structures métalliques. On retiendra deux noms, d'abord celui de Dupuy, qui, le premier, eut l'idée d'un appareil pour mesurer le travail du fer (1877), et celui de C. Rabut (1852-1925). Ce dernier, grâce à une campagne de mesure entre 1891 et 1895, a pu éviter le renforcement de la quasi-totalité des ponts du réseau de l'Ouest, en montrant que les liaisons hyperstatiques jusque-là négligées dans le calcul permettaient aux ponts de supporter le poids croissant des locomotives et des convois qui leur étaient attachés.
Dans le domaine des sols, un apport fondamental d'origine expérimentale est celui dû à Darcy (1803-1858) qui dans une note en annexe à son ouvrage ${ }^{(7)}$ sur l'alimentation des fontaines de Dijon, définissait le coefficient de perméabilité, la loi d'écoulement permanent et celle à charge variable (Fig. 4). Ce fut l'outil qui permit de modéliser l'écoulement de l'eau dans les sols.

La deuxième moitié du XIX ${ }^{e}$ siècle ne vit guère de progrès dans le domaine de la connaissance des argiles. La mise en évidence du rôle de l'eau, la conceptualisation de la notion de pression interstitielle ont exigé des observations et un apprentissage qui ont demandé un temps, qui ne devait porter ses fruits qu'au début du $\mathrm{xx}$ ' siècle avec Terzagihi.

Par contre dans les sables, Boussinesq (1842-1929), sur le plan theorique, établit, d'une part, les équations d'équilibre derrière un mur de soutènement avec frottement entre le massif et le mur, qui ne furent intégrées que par Caquot et Kérisel en 1934, et, d'autre part, les équations permettant de calculer contraintes et déformations dans un massif élastique. Son analyse de 1883 des essais de Darwin, qui śappuyait sur ses travaux de 1876, est un exemple remarquable d'analyse en retour. Cette approche était complétée par les essais de O. Reynold (1842-1912) sur la dilatance des sables (1887), qui permettait de mieux cerner le domaine de variation de l'angle de frottement d'un sable suivant les conditions de sollicitations.

17I Darcy G. - Les fontaines publiques de la ville de Dijon. Paris, Victor Dalmont. 1856.

$$
\text { Appareil dextinc a détermiase la loi }
$$

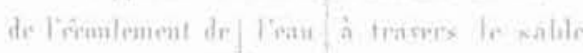

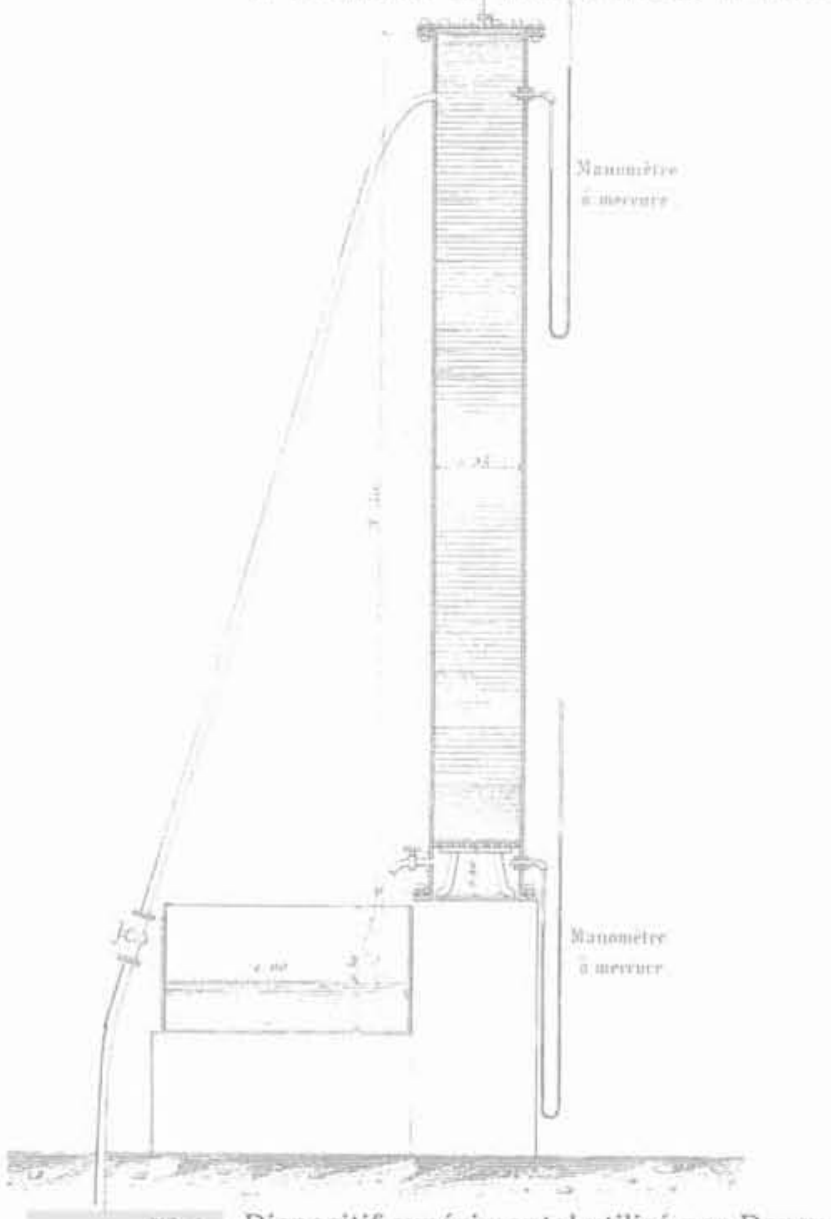

FG. 4 Dispositif expérimental utilisé par Darcy. Experimental apparatus used by Darcy. 


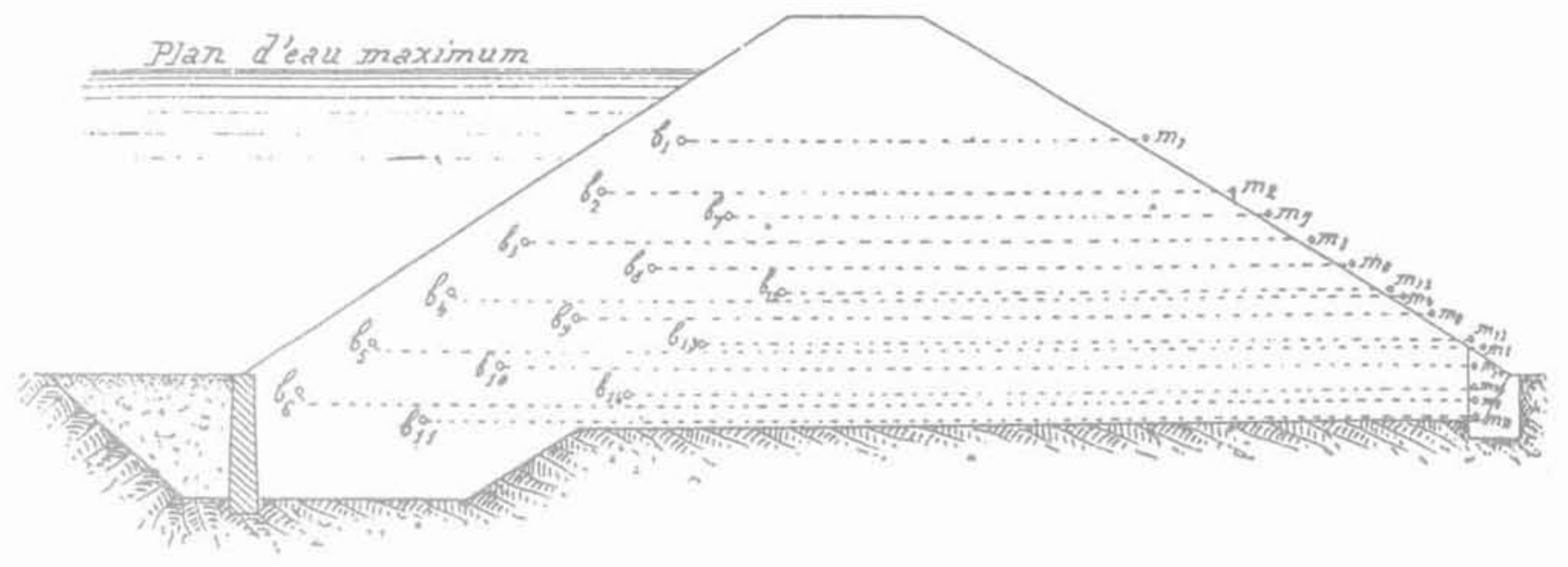

Fị, 5 Propositions pour l'observation des pressions hydrostatiques à lintérieur des massifs. Proposal for observation of hycirostatic pressures within earth embankment.

Le développement des travaux d'aménagement du territoire dans le domaine hydraulique, dans toute l'Europe ou dains les territoires sous domination européenne comme les Indes, amena des progrès en réponse à des questions liées à la sécurité des ouvrages. C'est le cas des mesures de pression sous les radiers de barrages dirrigation fondés sur du sable, conduites par J. Clibborn (1847-1938) et J. Beresford (1845-1927) sur des sites différents peu avant 1900, aux Indes. Ces essais fournirent des données pour comprendre les mécanismes d'érosion interne et de boulance, à partir de la connaissance de la répartition des pressions sous les radiers, et des remèdes pour lutter contre. Des nombreux ouvrages, construits aux Indes et en Egypte par la suite, Bligh allait tirer des règles de dimensionnement.

Les argiles allaient revenir au centre des préoccupations des ingénieurs pour les raisons déjă énoncées, relatives au développement des infrastructures. Dans le domaine de la caractérisation de la fraction argileuse, un pas essentiel a été franchi grâce à A.M. Atterberg (1846-1916), chimiste et spécialiste des sols, chercheur dans le domaine agricole en Suède. Il proposa et fit. adopter, en 1913, une classification fondée sur la dimension des particules et en particulier la valeur de $2 \mu$ pour les argiles, Mais, conscient de son insuffisance, Il y ajouta les notions de plasticité définie par la différence entre des teneurs en eau correspondant à la limite d'écoulement sous choc (liquidité) et celle d'émiettage (limite de roulement). Ces travaux furent publiés entre 1910 et 1913 . Ils passèrent assez inaperçus, mais Terzaghi les remarqua et les intégra dans son programme de recherches dès 1919.

C'est à partir du terrain que de nouvelles avancées se firent. En France, la digue de Charmes ${ }^{(B)}$ fut, à la suite de la rupture de son talus amont en 1909, l'occasion pour J. Frontard (1884-1962) de conduire une étude très complète du problème des accidents constatés sur les digues en France tout au long du XIX siècle, d'en chercher les causes et de proposer des remèdes. Il étudia l'influence du pourcentage d'argile dans les terres constituant les talus des différentes digues existantes, certaines ayant été affecté par des accidents d'autres non. Ensuite, sur la suggestion de Résal. Fron-

iin Frontard 3. - n. Notice sur l'accident de la dique de Charmes in Annales des ponts et chaussées, vol, 23, 1914 tard réalisa des essais de cisaillement, sur des échantillons constitués de matériaux de même nature que celle de la digue, de $0,77 \mathrm{~m}^{2}$, de $20 \mathrm{~cm}$ d'épaisseur, soumis à un double cisaillement. Ces essais, conduits sous différentes charges verticales, lui permirent de déterminer un angle de frottement. Il étudia ensuite quelle pouvait être l'influence de la proportion relative de sable et d'argile. Enfin, et ce n'est pas le moins remarquable, il pensait que la pression de l'eau entre les feuillets fonction de la charge régnant dans la retenue avait une importance mécanique, et suggérait au moyen d'un dispositif adéquat d'en mesurer la valeur en différents points (Fig. 5).

A la même époque fut relevée pour la première fois la surface de la nappe qui s'établit dans un barrage en terre, le barrage de Belle Fourche, aux USA, barrage homogène de 36 mètres de hauteur. Soixante-treize points de mesure constitués par des tubes en fer galvanisé, de 2 pouces de diamètre, ouverts aux deux extrémités furent implantés. Les niveaux d'eau furent mesurès depuis 1911 au moins jusqu'en 1924

A.L. Bell (1874-1956), dans un remarquable exemple d'aller et retour entre la théorie et la mesure en laboratoire, pour vérifier la validité de formule relative à la poussée des argiles et à leur force portante, imagina un appareil de cisaillement direct pour le laboratoire (1915), dont est issu celui que nous connaissons tous.

J. Résal (1854-1919), dans le deuxième tome de son traité sur la poussée des terres (1910), traitait des terres cohérentes, en s'appuyant sur des observations de terrain relatives à la résistance à la traction des argiles.

Un glissement en grand d’un mur de quai, à Göteborg, en 1916, permit à Petterson (1881-1966) de proposer une méthode de calcul de stabilité avec $\mathrm{c}=0$, suivant un cercle de rupture et un découpage de la masse ayant bougé en tranches verticales. Cette proposition fut reprise par Fellenius (1876-1957) pour l'étude de la stabilité de pentes argíleuse avec $\phi=0$, proposition faite pendant la guerre de 1914-1918 et publiêe en 1927. Entre 1913 et 1921, une prospection systématique de pentes instables, conduite à l'initiative des chemins de fer suédois, permit de mieux connaitre les propriétés des argiles et d'améliorer les moyens de déterminer leurs propriétés.

En même temps qu'il montrait l'importance de la cohésion et des caractéristiques élastiques des sols, Ter- 
zaghi ${ }^{19}$ (1883-1963) établissait que les sollicitations extérieures qui s'exercent sur un massif saturé d'eau se répartissaient entre le squelette, ou ensemble des grains constituant la partie solide du sol, et l'eau ou pression interstitielle. Seul le squelette constitué par les grains présente une résistance mécanique ou résistance au cisaillement pour équilibrer les sollicitations extérieures. Cette résistance sera d'autant plus réduite que la pression de l'eau sera plus élevée. Ce principe unificateur permit alors un développement sans précédent de la géotechnique. Il fưt énoncé explicitement en 1923 en relation avec le phénomène de la consolidation de l'argile. Le livre de Terzaghi, Erdbaumechanik fondait la mécanique des sols comme une discipline à part entière du génie civil. On peut voir dans l'apport de ce dernier aussi, un aboutissement de l'école expérimentale germanique.

\section{Le temps des laboratoires de mécanique des sols (après 1920)}

Les travaux de Terzaghi sur la consolidation des argiles ouvraient une ère nouvelle en apportant aux ingénieurs des outils théoriques qui leur permettaient enfin d'avancer dans la compréhension du comportement des sols, et plus précisément des argiles. Toutefois, cet apport ne pouvait être fécond que si sa mise en cuvre s'appuyait sur la méthode qui avait permis à Terzaghi, de progresser. Ce dernier écrivait en 1927 :

Les problèmes de fondations sont toujours tels qu'une approche strictement théorique et mathématique sera toujours impossible. La seule façon de les résoudre avec efficacité consiste tout d'abord à découvrir ce qui s'est passé dans des cas analogues, ensuite le type de sol concerné, et finalement, pourquoi les opérations ont conduit à certains résultats. En accumulant systématiquement un tel savoir, les données empiriques étant bien définies par une reconnaissance correcte des sols, le calcul des fondations pourrait se développer sous la forme d'une science semi-empirique.

\footnotetext{
in Skempton A.W. - "Terzaghi's discovery of effective stress in, in From theory to practice in soils mechanics fEd. Blemum. Casacrande, Skempton), New York. J. Wiley, 1960.
}

... L'essentiel de ce travail, l'accumulation systématique de résultats empiriques, reste à faire ${ }^{i r c h}$.

C'était une démarche identique à celle qu'avait suivie Collin, qu'il avait formulée presque dans les mêmes termes. Elle allait être menée sinon à son terme, du moins suffisamment loin pour que des progrès très importants puissent être enregistrés, dans les décennies qui suivirent. Il fallait, d'une part, multiplier les essais de laboratoire pour étudier le comportement des sols à petite échelle, d'autre part, faire des mesures à plus grande échelle sur les sols en place au moyen d'essais in situ, et enfin recueillir le résultat d'observations sur le comportement des ouvrages.

C'est dans cet esprit que fut créée entre 1920 et 1934, de façon institutionnelle, une grande partie des laboratoires de mécanique des sols (11). Vingt-cinq laboratoires, dont onze pour les États-Unis et quatre pour l'Allemagne rendaient compte de leur activité au Congrès de Cambridge en 1936. Le premier fut créé en 1920 aux États-Unis pour l'étude des routes (US Bureau of public roads). Suivit un laboratoire au Japon en 1923 , celui de Freiberg en Allemagne en 1924. Celui de Vienne en Autriche fut créé en 1929 par A. Casagrande au cours d'une mission organisée par Terzaghi, laboratoire dirigé par Léo Casagrande. Ensuite, les créations se succédèrent, celles du MIT et de l'université de Yale en 1930, de celle de Harvard 1932, de la Building Research Station à Londres en 1933, à la station d'essais de Vicksburg du corps des ingénieurs (WES) et au Bureau of Reclamation (USBR) en 1933, du CEBTP à Paris et de Delft aux Pays-Bas en 1934, de l'université

\footnotetext{
190 Terzaghi K. - a La science des fondations, Son état actuel, son ave-

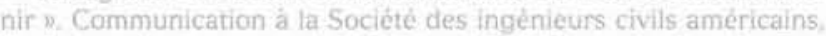
Technique des travaux, vol. 4 (1928), vol, 5 (1929), traduction des Proceedings ASCE, vol. 53 et 55,1929

na. Une section spéciale (section A volume 2) fut consacrée à la description des laboratoires existants leur historique leur équipement. dans les comptes rendus du premier congrès international de mécanique des sols et des fondations à Cambridge, sous le titre gènéral Reports from soils mechanics laboratories or testing apparatus technicque of testing and investigations in progress

La plupart des informations sur les appareils, mèthodes, procèdes sont issues des comptes rendus des deux premiers congres de mécanique des sols. des articies techaiques reiatifs à ces produits et enifin des traités et manuels de l'époque. Cette bibliographie érait trop importante pour figurer dans cet article.
}

TABLEAU Historique des essais in situ

\begin{tabular}{|c|c|c|}
\hline Nature ou designation de l'essal & Dates & Alifeurs oti promoteurs \\
\hline Esșai à la barre à mine & $\begin{array}{l}\text { Pratique immémoriale } \\
\text { AuXVII" siècle }\end{array}$ & Belidor \\
\hline Essai de chargement à là plaque & $1838 ?$ & A. Collin \\
\hline Standard penetration test (SPT) & $\begin{array}{c}1902 \text { (prise d'échantillons par battage) } \\
1920 \text { (mise au point de l'essai) } \\
1927 \text { (codification) }\end{array}$ & $\begin{array}{l}\text { C.R. Gow } \\
\text { L. Hart. G. Fletcher }\end{array}$ \\
\hline Essai de pénétration statique (CPT) & $\begin{array}{c}1917 \\
1927 \\
1935 \text { (brevet cóne mobile) }\end{array}$ & $\begin{array}{l}\text { Chemins de fer suédois } \\
\text { Chemins de fer danois } \\
\text { Barentsen }\end{array}$ \\
\hline Scissomètre (vane test) & 1948 & L. Carison \\
\hline Pressiomètre & 1954 & Ménard \\
\hline Pièzocone & $\begin{array}{l}1974 \\
1976\end{array}$ & $\begin{array}{l}\text { Janbu, Senesset } \\
\text { 1. Parez }\end{array}$ \\
\hline
\end{tabular}


TABLEAU \#I Historique des méthodes et matériels de sondages et de prélèvement

\begin{tabular}{|c|c|c|}
\hline $\begin{array}{l}\text { Nature ou désignation du matériel } \\
\text { ou des méthudes }\end{array}$ & Dates & Auteurs ou promoteurs \\
\hline Puits & $\begin{array}{l}\text { Pratique immémoriale } \\
\text { Au XVIII siècle }\end{array}$ & Délius, Perronet, Bossut \\
\hline Percussion & Pratique très ancienne, connue des Chinois & \\
\hline Tarière & $\begin{array}{l}\text { XIVe siècle } \\
\text { XVIt siècle }\end{array}$ & B. Palissy \\
\hline Rotation et couronne diamant & 1862 & G.A. Leschot \\
\hline Rotary & 1901 & J.-F. Lucas \\
\hline Caróttage ẻlectrique & 1927 & C. Schlumberger \\
\hline Tarière et lançage hydraulique & 1942 & O. Mohr (méthodologie) \\
\hline $\begin{array}{l}\text { Divers types de carottier pour sol meuble } \\
\text { à partir de } 1920 \\
\text { Carottiers à piston }\end{array}$ & $\begin{array}{l}1920 \\
1948\end{array}$ & $\begin{array}{l}\text { Carottier de Mohr } \\
\text { Hvorslev (condition de mise en ceuvre } \\
\text { des carottiers à mince paroi) }\end{array}$ \\
\hline Canottier Denison & 1940 & Johnson \\
\hline
\end{tabular}

de Cormell en 1935. Bien sûr cetté énumération n'est pas exhaustive.

Il n'est pas dans notre propos de retracer tous les progrès de la mécanique des sols après la Seconde Guerre mondiale. Nous souhaitons simplement, dans la continuité du regard que nous avons porté sur le passé, montrer la rupture intervenue non dans la nature des recours à l'expérience, mais dans la quantité des outils mis à la disposition des ingénieurs, et celle des informations qui ne pouvaient qu'en résulter. Le développement des moyens de calcul, à partir des années 60 , va permettre de traiter les informations obtenues ou de les utiliser dans le cadre d'une modélisation sans précédent jusqu'alors.

Mais il n'est pas inutile d'insister sur la difficulté, pour une communauté technique, de faire sienne une avancée technique. Bien que Terzaghi eut de façon très claire précisé la notion de pression interstitielle dans des articles en 1924,1927, 1932, et 1936, on peut lire sous la plume de plusieurs ingénieurs du Bureau of Reclamation que ces derniers considérèrent au début la notion de pression interstitielle comme un phénomène de laboratoire, et n'en tinrent compte pour expliquer des instabilités de pentes qu'à partir des années 1937 à 1938 ,

Les tableaux qui suivent montrent l'évidente corrélation entre la mise en place des structures et le développement des moyens de reconnaissance et de mesure dont disposèrent les ingénieurs en moins de trente ans, et ceci malgré la Seconde Guerre mondiale.

Pour deux des plus importants essais in situ, on a indiqué plusieurs dates et auteurs ou promoteurs, pour souligner le caractère très progressif des avancées et la succession des intervenants dans des pays différents, soulignant par là même le caractère collectif de ce travail dú à l'extrême complexité du réel.

Le développement de la mécanique des sols, après 1920, la concomitance avec celle des grands ouvrages hydrauliques n'étant pas un hasard, a exigé de son côté la mise en ceuvre de techniques de prélèvements de roches meubles qui perturbent le moins possible le sol, pour pouvoir réaliser les essais qui s'imposaient pour caractériser les sols.

Le développement de la pratique des sondages dans le génie civil est dü dans un premier temps à la construction des grands barrages à la fin du XIX $\mathrm{X}^{\varepsilon}$ et au début du XXe siècle. Les sondages avaient été surtout utilisés pour trouver de l'eau et en particulier en exploitant les nappes artésiennes, dès la fin du XVII'. On citera les noms de Mullot (1792-1872) qui après dix ans d'effort parvint à $500 \mathrm{~m}$ de profondeur en 1843 à Grenelle, de Degoussée et enfin de Lippman.

A Serre-Ponçon entre 1899 et 1925 et à Génissiat à partir de 1910, les moyens des soncleurs étajent encore très limités. Le procédé de perforation par rotation avec des couronnes diamant, fut mis au point avec beaucoup de difficulté à cause du problème de fixation des diamants. Ce n'est qu'après 1920, que les performances des moyens de reconnaissance furent sensiblement améliorées. La construction de très nombreux barrages en particulier aux États-Unis et dans toute l'Europe, est certainement une des raisons des progrès effectués, qui se sont appuyés par ailleurs sur les moyens mis en ceuvre dans la prospection pétrolière et minière.

Enfin, le dernier tableau donne les dates à partir desquelles commença la mise au point des matériels d'auscultation, mise au point souvent difficile.

Le tableau suivant donne la chronologie des essais de sol qui simultanérnent utilisèrent les échantillons prélevés.

Nous terminerons cet historique sur quelques mots concernant la description des sols, dont la codification a beaucoup d'importance pour la transmission de l'expérience. Casagrande disait que des deux chapitres de la mécanique des sols les plus sujets à discussion, si celui de la résistance au cisaillement des sols est le plus difficile, celui de la classification des sols est le plus confus ${ }^{112 !}$. A partir de la notion de courbe granulométrique, et des notions développées par Atterberg, les

\footnotetext{
(12) Casagrande A. $-\pi$ Classification and identification of soils ». ASCE
} Transactions, vol. 113, 1948 
TABLEAU iII Historique des méthodes et matériels de laboratoire

\begin{tabular}{l|c|c}
\hline Nature ou désignation des appareils & Dates & Auteurs ou promoteurs \\
\hline $\begin{array}{l}\text { Équipement pour l'analyse } \\
\text { de la fraction argileuse }\end{array}$ & 1932 & A. Casagrande \\
\hline $\begin{array}{l}\text { Coupelle pour la détermination } \\
\text { de la limite de liquidité }\end{array}$ & 1932 & A. Casagrande \\
\hline $\begin{array}{l}\text { Appareil de cisaillement cirect } \\
\text { Appareil de chargement pour }\end{array}$ & 1934 (normalisation) & A.t. Bell \\
Iétude de la consolidation & 1919 & A. Casagrande \\
\hline $\begin{array}{l}\text { Cellule de chargement triaxial } \\
\text { Première mesure de pression } \\
\text { interstitielle }\end{array}$ & 1936 (normalisation) & A. Casagrande \\
\hline $\begin{array}{l}\text { Appareil de cisaillement } \\
\text { par torsion circulaire }\end{array}$ & 1932 & $\begin{array}{l}\text { Casagrande } \\
\text { USBR } \\
\text { Bishop et Henkel (traité) }\end{array}$ \\
\hline
\end{tabular}

TABLEAUIV Historique des matériels d'auscultation

\begin{tabular}{|c|c|c|}
\hline Nature du materriel ou des méthodes: & Dates & Anteurs ou prompteurs \\
\hline Relevé topographique & $\begin{array}{l}\text { Structures, début XIX: siècle? } \\
\text { Barrages, Grosbois } 1852\end{array}$ & \\
\hline Auscultation des structures métalliques & $\begin{array}{l}1877 \\
1890\end{array}$ & $\begin{array}{l}\text { Dupuy } \\
\text { C. Rabut et Manet }\end{array}$ \\
\hline $\begin{array}{l}\text { Relevé de niveau de nappe } \\
\text { dans un barrage en terre. }\end{array}$ & $\begin{array}{l}\text { Début XIX siècle? } \\
\text { Belle Fourche } 1911 \\
1933\end{array}$ & $\begin{array}{l}\text { USBR } \\
\text { USBR (WLI) }\end{array}$ \\
\hline Cellule à contre-pression & $\begin{array}{l}1916 \\
1938 \\
1958\end{array}$ & $\begin{array}{l}\text { Goldbeck } \\
\text { USBR (HPI) } \\
\text { F. Glötzl }\end{array}$ \\
\hline $\begin{array}{l}\text { Extensomètre à résistance tendue } \\
\text { Cellule Carison }\end{array}$ & $\begin{array}{l}1925 \\
1930 \\
1952\end{array}$ & $\begin{array}{l}\text { Carison } \\
\text { Carison }\end{array}$ \\
\hline Extensomètre à corde vibrante & 1930 & A. Coyne \\
\hline Piézomètre ouvert & 1949 & A. Casagrande \\
\hline Cellule à jauges & 1955 & W.E.S. \\
\hline Plézomètre hydraulique & $\begin{array}{l}1939 \\
1960 \\
\end{array}$ & $\begin{array}{l}\text { USBR } \\
\text { Bishop }\end{array}$ \\
\hline Piézométre à corde vibrante & 1958 & Télemac J. Bellier \\
\hline
\end{tabular}

movens offerts par les laboratoires pour mesurer ou établir les caractéristiques correspondantes ont permis une description rationnelle et une classification, qui seules permettaient la transmission des données de l'observation, et qui constituait l'outil à la base de bien des corrélations. Feld, dans un article sur les oriqines de la mécanique des sols publié dans les actes de la conférence de Rotterdam, en 1948, consacre un paragraphe à ce problème. Il cite à cet effet plusieurs auteurs, depuis Evelyn qui exposait la question, en 1675, devant la Royal Society, jusqu'à Humphrey David qui traitait ce problème du point de vue de l'agriculture en 1813. Il citait au passage les contributions de Lemaire (1737), Querlonde (1743), Chauvelot (1783). Toutes les définitions s'appuyaient sur une description naturaliste et laissaient une grande part d'interprétation à ceux qui voulaient s'en servir.
Skempton rappelle la description de Bélidor. accompagnée de valeurs de poids volumique, distinguant rocher, sable et terre, qui lui semble la première et la plus complète de celles du xvine siècle.

Nous avons recherché tout au long du XIXe siècle quelles pouvaient être les données de référence sur les sols. L'absence de références chiffrées et de mode de leur obtention est certainement une des explications des réclamations sans fin des entrepreneurs et des très nombreuses ruptures de marché. Il était dès lors très difficile de juger de la validité des récriminations tant de la part de l'entrepreneur que du maitre d'ouvrage. Par exemple la granulométrie des sables criblés depuis des temps immémoriaux n'a été quantifiée que lors des études sur le béton de Féret (1892).

De nombreuses classifications granulométriques y compris pour la partie arqileuse furent proposées après 1920. Mais c'est à partir de l'entrée en guerre des États- 
Unis, pour juger de l'aptitude d'un site à la construction d'un terrain d'aviation que Casagrande élabora ce qui devait servir au cadre d'une classification unifiée des sols en intégrant les données granulométriques, les limites d'Atterberg, et en définissant des pratiques d'essais en laboratoire et des procédures d'identification sommaire sur le terrain.

\section{Remarque sur une exception française}

Le développement de tous les matériels de prélèvement, de mesures in situ ou en laboratoire souvent imparfaits, mais qui avaient le mérite d'orienter ou d'appuyer le travail créatif de l'ingénieur, eut pour conséquence de créer un climat propice au travail d'innovation des procédés de construction. C'est tout un chapitre de la mécanique des sols appliquée, qu'il convient de ne pas passer sous silence, à défaut de pouvoir le traiter en détail dans le cadre de cet article. I] relève plus peut-être qu'un autre de l'approche expérimentale de la mécanique des sols.

Ce sont les entrepreneurs français qui y furent particulièrement brillants pour tous les procédés relevant de l'injection et des fondations spéciales. En fait, c'était une tradition qui remontait au milieu du XIXé siècle, qui résultait de la conjonction de deux condítions particulières propre à l'espace technique et économique français. L'entrepreneur français s'est trouvé très vite confronté à un marché étroit dans lequel la compétition était très dure. Il trouvait en face de lui un client qui était à la fois maitre d'ouvrage et maitre d'ceuvre, qui n'a pas dédaigné d'écouter ses propositions d'amélioration des projets qui avait été établis par ses propres services. L'innovation s'imposait comme moyen d'augmenter ses marges ${ }^{\text {(153) }}$

La confusion de la fonction de maitre d'ouvrage et de maitre d'ceuvre, particulière à la France, avait pour origine le fait que les grands travaux pour l'essentiel étaient financés ou garantis par l'État, au contraire de ce qui se passa en Angleterre. On comprend que l'État ne pouvait pas confier à un tiers la conception et la gestion des travaux de construction des fortifications, d'arsenaux, des routes qui les desservaient, des canaux, dont il avait conçu le réseau, en vue d'aménager le territoire suivant une vision qui lui était propre.

Nous voyons dans la présence, sur ordre de Colbert, du chevalier de Clerville (1610-1677), commissaire général aux fortifications prédécesseur de Vauban, auprès de Riquet qui ne pouvait financer que $20 \%$ de la dépense du canal du Midi, le début d'une pratique qui relève de l'exception française, et qui s'étendit sur plusieurs siècles. C'est le chevalier de Clerville qui établit le devis du canal, c'est-à-dire au sens du XVII siècle, le projet. C'est ainsi qu'il proposa la réalisation d'un réservoir d'alimentation unique au site de Saint-Ferréol, au lieu d'une dizaine de réservoirs comme pour le canal de Briare ${ }^{\text {it4 }}$.

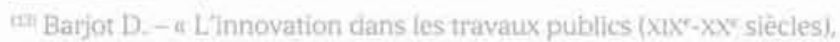
une réponse des firmes au défi de la demande publique $n$. Histoire, èconomie et société, $n^{\circ} 2.6^{\prime}$ année. 1987.
}

w1 Gazelle F, - Riquet et les eaux de la montagne noire, l'idée géniale de l'alimentation du canal n, dans Histoire du canal du Midi. Edition du tricentenaire sous la direction de J.D. Bergasse, vol. 4, 1985, p. 145. 169

Voir aussi tome 3, Blanchard A, Bergasse J.D. - $\alpha$ Ingénieurs du Boy et canal des deux mers is.
L'expertise technique des services de l'État permettait un dialogue fructueux avec les entrepreneurs, facilité par la possibilité de passer des marchés sur concours (1882), d'autant plus que les frontières entre entrepreneurs et Administration étaient franchies facilement avec une pratique très libérale des congés. Il faut y voir l'origine des compétences très étendues des entrepreneurs français, qui au cours du XIXe brillèrent déjà dans les travaux portuaires et dans les fondations à l'air comprimé ${ }^{175}$. Ces compétences aidèrent à la réussite à l'exportation de la profession. En 1913, les dix plus grandes entreprises françaises (SGE, GTM, Hersent, Fougerolle, entre autres), réalisaient $60 \%$ de leur chiffre d'affaires à l'exportation (1e. La mondialisation qui nous concerne tant aujourd'hui, était alors aussi avancée que dans les années 70 .

Les sociétés d'électricité, fondées à la fin du $\mathrm{XIX}$ siècle, créèrent également les conditions d'une liberté qui facilita les initiatives, et une créativité que ne permettait pas la lourdeur d'un service d'État. Une partie de la communauté technique française trouva en Algérie des conditions géomorphologiques qui l'obligea à des solutions originales, en particulier pour tout ce qui touchait au sol. Sa réussite fut connue des EtatsUnis. Elle y trouva aussi la solution de l'injection des alluvions ${ }^{\mid 11}$. Ces pratiques furent continuées par les grandes entreprises publiques après la Seconde Guerre mondiale.

\section{Les échanges et transferts technologiques}

L'exposé sommaire qui vient d'être fait montre que, dans l'activité du génie civil et en mécanique des sols plus particulièrement, les échanges et transferts technologiques sont inhérents à la pratique de ce qui reste par bien des aspects un art. On peut distinguer comme canaux des échanges technologiques, les hommes, les structures comme les Académies et les sociétés savantes, les livres techniques, les revues, les missions et, enfín, les congrès. On se propose d'évoquer quelques cas pour chacun des aspects ainsi dénombrés.

Les techniciens ont voyagé à toutes les époques et depuis les plus anciennes. Ils furent les vecteurs des échanges soit qu'ils s'installent dans un autre pays que le leur, soit qu'ils viennent pour des périodes plus ou moins longues, ingénieurs hollandais pour apprendre aux Français les techniques d'assèchement, ingènieurs du Harz pour les mines, les Italiens pour lírrigation en Provence, et beaucoup d'autres. Lorsque Francois Jer ramena Léonard de Vinci d'Italie, c'était d'abord un ingénieur qui venait avec toute l'expérience de l'Ttalie dans le domaine des canaux. La première idée du canal du Midi lui est due, en réponse au problème du trans. port qui lui avait été posé par le pouvoir royal.

Les institutions comme les Académies et équivalentes furent des foyers d'échanges dès leur création.

\footnotetext{
14 Glossop R - \& The invention and early use of compressed air to exclude water from shafts and tunnels during construction n. Gêo. technique, vol. 26, $\mathrm{n}^{\circ}$ 2. June 1976, p, 253-280.

14it Barjot D, - w La grande entreprise de travaux publics (1883-1974), contraintes et stratêgie n. Thèse de doctorat d'Etat. Paris IV-Sorbonne. 1989

177 Glossop R. - u The invention and development of injection pro. cess 3. Part I, 1802-1850, Géotechnique, vol. 10, sept. 1961. Part II. 18501960), Gêntechnique, vol. 11. dec 1960
} 
Belidor fut Fellow of the Royal society de Londres, consacrée aux sciences et fondée en 1662, comme Perronet ultérieurement. L'Académie des sciences à Paris fut fondée en 1666 . Elle comprenait des membres étrangers comme Huyghens. D'autres capitales se doterent d'institutions analogues, les capitales provinciales françaises également.

Des sociètés pour favoriser le dẻveloppement scientifique à des fins industrielles ou furent fondées, comme la Société pour l'encouragement à l'industrie nationale (SEIN) en 1802 à Paris puis à Mulhouse en 1827 . Puis vinrent les sociétés savantes, celle des ingénieurs civils anglais en 1818 , la Société de géologie à Londres 1820, à Paris 1830, la Société des ingénieurs civils à Paris 1848, pour n'en nommer que quelques. unes. Toutes ces sociétés, comme les Académies, avaient des bulletins ou des recueils des mémoires acceptés par leurs instances.

Les transmissions du savoir étaient fonction des supports matériels de l'époque. Au XVII' siècle les revues étaient exceptionnelles, mais les livres sans être aussi abondants qu'au XIX' siècle, existèrent en nombre conséquent à partir du début du XVIII sjècle fGautier. Belidor, Dubuat, Bossut, Lalande, Gauthey, Girard). Les traductions étaient choses ordinaires, Delius, Silberschlag, auteurs allemands, furent traduits, mais aussi des auteurs anglais, italiens. Les traductions se faisaient dans les deux sens, même si la pratique du français était très répandue dans les classes aisées de toute l'Europe. Le mémoire de Coulomb fut traduit en allemand par Woltman en 1790, les aides-mémoires de Morin en anglais pour les Ettats-Unis où le corps des ingénieurs à ses débuts utilisa les manuels français de Navier.

Le nombre de livres publiés augmenta tout au long du XIX siècle. Mais le phênomène nouveau fut celui de la multiplication des revues. Bruno Jacomy rapporte qu'il y avait dix périodiques scientifiques et techiniques à la fin du XVII" siècle, cent au début du XIX et dix mille vers 1900.

En France, dans le domaine du génie civil, deux revues méritent une mention spéciale, pour le $\mathrm{XIX}$ siècle, les Annales des ponts et chaussées, fondèes en 1830, et le Génie civil, fondé en 1880. La prenière, véritable organe du corps des ponts et chaussées, portait à la connaissance de ses abonnés toutes les avancées ou réalisations dans le domaine qui était le sien avec une périodicité trimestrielle. La revue du Génie civil, devenue très rapidement hebdomadaire, était plus ouverte sur l'ensemble des techniques. Il faut dire que celle-ci a été créée à un tournant du siècle quí marquait, avec l'irruption de l'électricité, le début de la deuxième révolution industrielle. Ces deux revues étaient extrêmement ouvertes sur le monde, et contenaient des comptes rendus abondants des lives et publications étrangères ainsi que des comptes rencus de visites ou de missions.

Un autre canal de transfert technique est celut des missions d'études. Les missions de visite en GrandeBretagne et aux États-Unis se succédèrent sans discontinuer tout au long du XIX* siècle, dès la fin des guerres napoléoniennes. Mais elles ne se limitèrent pas à ces seuls pays, elles se firent partout où il y avait quelque chose à apprendre, comme les irrigations en Italie. A des fins différentes, les séjours d'assistance technique des ingénieurs du corps des ponts furent fréquents (Brésil, Pérou, Russie, Europe centrale, Turquie
Égypte). Cinq pour cent du corps étaient détachés à cet effet vers 1873 .

Vint ensuite le temps des congrẽs qui se développèrent dans un premier temps à l'ombre des expositions. universelles, dont la première est celle de Londres en 1852 , suivie de cinquante-neuf autres jusqu'en 1920. D'autres expositions internationales mais spécialisées comme celle de 1881 à Paris, consacrées à l'électricité, marquèrent leur époque.

Le premier congrés de génie civil eut lieu à Paris en 1880, celui de la navigation intérieure en 1885 à Bruxelles, celui de l'association internationale pour I'essai des matériaux en 1889 quí en 1901 tenait à Budapest son quatrième congrès.

Dans les années 1880, début de la seconde révolu: tion industrielle, une accélération dans le rythme des échanges se fit sentir. Au congrès de navigation intérieure de Paris en 1892, il y avait 1472 délégués venant de 22 pays différents. Ces réunions furent des occa. sions de rencontres au cours desquelles se tissèrent toujours plus nombreux des liens personnels entre hommes de métier, qui facilitèrent l'échange d'expérience.

Au fur et à mesure, de nouveaux congrès internationaux furent institués pour répondre au besoin d'approfondissement dé la connaissance, celui de la Houille Blanche en 1902, de la Poute en 1908, des Grands Barrages en 1933. Le premier congrès de la Mécanique des sols, à Cambridge, en 1936, s'inscrivait dans une évolution tout à fait normale. II réunit 206 participants:

Parmi les techniques ou procédés, objets de transfert technologique dans des domaines connexes à la mécanique des sols, on peut citer le barrage en maçonnerie moderne, dont le Furens (1862-1866), près de Saint-Étienne, fut le prototype. Appelé le barrage français, il servit de modèle dans le monde entier. A T'inverse, on remarquera le transfert des États-Unis vers la France, des techniques du barrage en terre et la trés grande vitesse à laquelle l'assimilation en fut faite. apres la Seconde Guerre mondiale

\section{Conclusion}

Cette histoire r'a pas de conclusion, puisqu'elle se continue sous nos yeux, certains d'entre nous y étant particulièrement actifs. Toutefois, après ce survol très rapide et forcément réducteur, il n'est pas inutile d'insister sur certain traits que nous n'avons peut-être pas assez soulignés.

Le gênie civil et la mécanique des sols quî fait partie intégrante du premier, ont été élaborés á partir d'une pratique expérimentale. Ceci ne veut pas dire que l'on peut se dispenser, ou tenir pour négligeable l'approche théorique, Cette dernière permet peut-être seule de dépasser, de conduire et d'organiser l'étude expérimentale. Mais celle-ci doit «faire apercevoir, démêler et déterminer les principes eux-mèmes, ou les lois scientifiques, les bases scientifiques... de l'établissement des équations qui en seront l'expression (17il.... n. Elle est au départ de tout progrès.

Tout ce travail trouve sa justification dans la réalisation des projets dont le comportement prime toute

\footnotetext{
n* Barré de Saint-Venant - " Sur le but thèorique des principaux tra vaux de Henri Tresca u. Comptes rendus de l'Académie des sciences 73 juillet 1885
} 
autre approche et celle du calcul en particulier. Ceux-ci ne sont pas commandés par une spéculation gratuite mais par la satisfaction des besoins de la société de l'époque, besoins dont l'économie n'est pas la seule raison. Les ingénieurs ne font que répondre à un cléfi permanent imposé par la demande publique ou privée.

Le champ du génie civil n'est pas celui de l'invention mais de l'tunovation, celle de matériaux (ciment, béton, béton armé et précontraint), de procédés (l'injection, les fondations spéciales, la terre armée), d'ouvrages (barrages en terre, barrages-voutes). II est un lieu de synthèse de tous les moyens du syslème technique d’une époque donnèe.

Si la communauté technique française a joué un rôle éminent pendant près de deux siècles dans ce domaine, au point d'être considérée par un historien anglais comme la patrie du génie civil, c'est parce que l'espace français politiquement unifié plus tột que bien d'autres pays en Europe, et qui était aussi le plus vaste, avait besoin đ'être défendu, et aménagé. Au début du $\mathrm{XX}^{\circ}$ siècle, la mise en valeur de l'Ouest américain eut des conséquences identiques dans le développement de la mécanique des sols pour les États-Unis.

Mais, si cette même communauté a su si bien remplir sa mission, c'est qu'elle y avait été très bien préparée. Dès la fin du xvilie siècle, il y avait en France cing écoles d'artillerie et trois écoles d'hydrographie. Ensuite, au XVII' siècle, la France a su se doter d'écoles dans le génie civil et les domaines connexes bien avant les autres pays. On se rappelle tous de l'École des ponts et chaussées fondée en 1747 ou de I'École polytechnique en 1793, ou de l'École centrale en 1829. On oublie plus facilement l'École des arts et métiers en 1780, des mines de Paris 1784, celle de Saint-Étienne 1816, I'Ecole d'artillerie de Metz en 1804.

Coulomb était l'élève de l'Écolè du génie de Mézières, fondée en 1748 . On a cité le nom de l'abbé Bossut qui fut son professeur, un autre maitre dans ce mème établissement s'appelait Monge

Cette leçon de l'importance de la formation des hommes est toujours actuelle.

\section{DENEBOLA-LCPC}

Un logiciel de calcul des écrans de soutènement (rideaux de palplanches, parois moulées) par la méthode du coefficient de réaction

Basé sur la méthode de calcul aux coefficients de réaction. DENEBOLA-LCPC permet de déterminer de manière rapide et aisée les efforts et les déformations dans un écran de soutènement fiché dans le sol, aux différentes phases de travaux et d'exploitation. L'inertie de l'écran peut ètre variable et il peut être soumis à: - des efforts de flexion en tête et à la base et, plus généralement, à des conditions aux limites les plus diverses: - des efforts hydratiliques dus aux pressions intersttielles

- l'action de tirants (précontraints ou non) et butons: - des moments extérieurs imposés:

- des déplacements imposés.

La méthode repose sur une modélisation simplifiée de l'action du sol sur l'écran dans laquelle l'action du sol sur l'écran est réduite, en chaque point de l'ouvrage, à gauche et à droite, à une pression horizontale, liée au déplacement du niveau considéré par une loi cle réaction. Les lois de réaction utilisées couramment, mais abusivement désignées par le terme de alois élastoplastiques $n$, sont des lois de mobilisation linéaire avec paliers de poussée et de butée. Elles sont donc typiquement composées de trois parties:
- le domaine de poussée limite caractérisé par la pression p.

- le dómaine de butée limite caractérisé par la pression $P_{p}$ :

- le domaine pseudo-élastique, caractérisé par le coefficient de réaction $k_{n}$; ce domaine peut lui-même être décomposé en sous-domaines, caractérisés par une succession de valeurs de $k_{h}$.

Initialement développé par le Laboratoire central des ponts et chaussées, ce logiciel a été mis à jour et doté de nouvelles fonctionnalités à l'occasion d'une évolution visant à offrir une interface utilisateur plus conviviale, fonctionnant sous Windows $9 x$ ou Windows NT.

Prix de lancement jusqu'au 20 décembre 2000 20000 F HT (23 920 F TTC) Distribuse pat

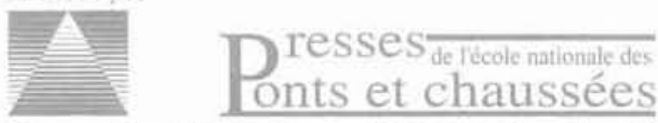

28, rue des Saints-Pères - 75343 Paris cedex 07 Tél : $33(0) 144582740$ - Fax: $33(0) 144582744$ e-mail : presses.ponts@mail.enpc.fr 